\title{
Patient perceptions of a comprehensive telemedicine intervention to address persistent poorly controlled diabetes
}

This article was published in the following Dove Press journal:

Patient Preference and Adherence

3 March 2017

Number of times this article has been viewed

\author{
Sara M Andrews' \\ Nina R Sperber ${ }^{1,2}$ \\ Jennifer M Gierisch ${ }^{1,2}$ \\ Susanne Danus' \\ Stephanie L Macy' \\ Hayden B Bosworth ${ }^{1,2}$ \\ David Edelman ${ }^{1,2}$ \\ Matthew J Crowley ${ }^{1,3}$ \\ 'Center for Health Services Research \\ in Primary Care, Durham Veterans \\ Affairs Medical Center, ${ }^{2}$ Division \\ of General Internal Medicine, \\ Department of Medicine, ${ }^{3}$ Division \\ of Endocrinology, Diabetes, and \\ Metabolism, Department of Medicine, \\ Duke University, Durham, NC, USA
}

Objective: We studied a telemedicine intervention for persistent poorly controlled diabetes mellitus (PPDM) that combined telemonitoring, self-management support, and medication management. The intervention was designed for practical delivery using existing Veterans Affairs (VA) telemedicine infrastructure. To refine the intervention and inform the delivery of the intervention in other settings, we examined participants' experiences.

Methods: We conducted semistructured interviews with 18 Veterans who completed the intervention. We analyzed interview text using directed content analysis and categorized themes by hemoglobin A1c (HbA1c) improvement $(<1 \%$ or $\geq 1 \%)$.

Results: Participants generally reported greater awareness of their blood glucose levels; however, they described dissatisfaction with the telemonitoring interface and competing demands during the intervention. Participants with $<1 \% \mathrm{HbA} 1 \mathrm{c}$ improvement reported that these challenges interfered with their engagement. Participants with $\geq 1 \%$ HbA1c improvement reported new self-management routines despite challenges.

Conclusion: Despite competing demands and frustration with the telemonitoring interface, many participants demonstrated intervention engagement and substantial improvement in HbAlc $(\geq 1 \%)$. Differences in engagement may reflect differing capacity to manage treatment burden. Because it relies on existing infrastructure, this intervention is a promising model for addressing PPDM within VA. Future work should focus on optimizing systems' telemedicine infrastructure; while reliance on existing infrastructure may facilitate practical delivery, and it may also limit intervention engagement by excessively contributing to treatment burden.

Keywords: telemedicine, persistent poorly controlled diabetes, type 2 diabetes, patient perspectives, treatment burden

\section{Introduction}

The complications and costs of diabetes rise exponentially as hemoglobin A1c (HbA1c) increases. ${ }^{1-3}$ Because HbA1c reduction prevents diabetes complications, ${ }^{1,4}$ patients with persistent poorly controlled diabetes mellitus (PPDM), or maintenance of an $\mathrm{HbA} 1 \mathrm{c}>9 \%$ for $>1$ year, despite clinic-based care, ${ }^{5}$ contribute disproportionately to the nationwide diabetes burden. ${ }^{6,7}$ The prevalence of PPDM is $\sim 10 \%$ of US adults with diabetes. ${ }^{6,8,9}$

Clinic-based care is suboptimal for PPDM patients because the infrequent patientprovider contact achievable in the outpatient setting may not support patients in accommodating the complex self-management demands PPDM requires. ${ }^{8,10,11}$ The burden imposed by PPDM is substantial; PPDM patients are expected to check their blood sugar several times daily, administer multiple daily insulin injections, modify
Correspondence: Matthew J Crowley Center for Health Services Research in Primary Care, Durham Veterans Affairs Medical Center, 508 Fulton Street (I52), Durham, NC 27705, USA

Tel + I 919286041 I ext 4066

$\mathrm{Fax}+19194165836$

Email matthew.crowley@va.gov
Patient Preference and Adherence 2017:II 469-478

469 
their diet, and exercise regularly. Concurrently, patients with PPDM often experience challenges that affect their capacity to manage this burden of demands; these challenges may include aversion to needles, ${ }^{12}$ comorbid conditions with complex treatment regimens, ${ }^{8,12}$ suboptimal patient-provider relationships, ${ }^{13}$ and deficits in knowledge, skills, motivation, social support, and self-efficacy. ${ }^{12-16}$ Patients with PPDM also face barriers unrelated to their disease that could impact their capacity to self-manage, such as work and family responsibilities. In the absence of additional supports to overcome these barriers, patients may be unable or unwilling to fully engage with diabetes self-management. ${ }^{17,18}$

Telemedicine is the remote delivery of health care services and clinical information using telecommunications technology. ${ }^{19}$ It is a modality for providing additional support to PPDM patients beyond what is feasible in the clinic setting. Specifically, telephone-delivered care can minimize treatment burden by eliminating some clinic appointments in favor of a more convenient mechanism. Studies have shown that provider review of self-monitored blood glucose (SMBG) data, self-management support, and medication management, all strategies that address specific factors contributing to poor diabetes control, can be adapted to the telemedicine setting and may even be enhanced by use of telemedicine. ${ }^{20-25}$ Telemonitoring, in which patients transmit SMBG values to providers via telephone, increases providers' access to data that can inform treatment recommendations. ${ }^{26}$ Both self-management support and medication management rely on regular, supportive interactions between patients and providers, ${ }^{10,11,21,26-28}$ which can be achieved more frequently and at lesser burden to patients when carried out via phone. Thus, a comprehensive telemedicine intervention combining telemonitoring, selfmanagement support, and medication management could address multiple factors underlying poor control and improve outcomes in the high-risk PPDM population.

However, few health care systems have implemented comprehensive telemedicine interventions for PPDM in clinical practice, potentially due to the fact that such interventions have seldom been designed for delivery under real-world conditions. ${ }^{29,30}$ If an intervention combining telemonitoring, self-management support, and medication management could be delivered using the target organization's existing staffing and infrastructure, it may be well suited to address the complex needs of patients with PPDM in practice.

Advanced Comprehensive Diabetes Care (ACDC) is a comprehensive telemedicine intervention for PPDM that was developed to 1) meet the complex management needs of patients with PPDM and 2) leverage existing telemedicine infrastructure within the Veterans Affairs (VA) health system. ACDC, which is designed for delivery by VA home telehealth (HT) nurses during biweekly telephone encounters, combines telemonitoring, self-management support, and medication management. While these intervention components have been individually found to improve glycemic control, bundling them into a comprehensive telemedicine intervention designed for practical delivery via existing infrastructure represents a novel approach. We piloted ACDC within the Durham VA during 2013-2014 and found that it improved $\mathrm{HbA} 1 \mathrm{c}$ by an average of $1 \%$ relative to usual care at 6 months. ${ }^{5}$ Patients completing at least half of the scheduled telephone encounters achieved a mean HbA1c reduction of $\sim 2 \%$. $^{5}$

To inform refinement and expansion of the ACDC approach, we qualitatively evaluated how, and for whom, ACDC worked to improve diabetes control. We conducted and analyzed semistructured interviews with participants to elicit their experiences with ACDC. We organized participant responses according to their clinical outcomes, specifically noting differences between participants with highest and lowest $\mathrm{HbA} 1 \mathrm{c}$ change ( $<1 \%$ vs $\geq 1 \%$ ). We also conducted interviews with the two VA HT nurses who delivered ACDC, to triangulate their accounts with those of participants.

\section{Methods}

Details of the ACDC randomized trial (NCT01778751) are described elsewhere. ${ }^{5}$ This study was approved by the Durham VA Medical Center Institutional Review Board. Briefly, ACDC was a 6-month telemedicine intervention $(\mathrm{N}=50)$ in which Durham VA HT nurses working with our research team delivered intervention content to study participants via biweekly calls. Participants were instructed to monitor their blood glucose three to four times per day and self-report their values daily using standard Durham VA telemedicine equipment (Cardiocom; TeleResponse Interactive Voice Response System, Chanhassen, MN, USA). During each call, HT nurses and participants reviewed interim blood glucose data, reconciled medications, and assessed medication adherence; at most encounters, nurses delivered self-management support modules on topics such as managing hypoglycemia. Following each encounter, a study physician reviewed patients' blood glucose data and recommended medication changes as indicated, and HT nurses implemented these recommendations.

At the end of the 6-month pilot, semistructured interviews were conducted with 18 out of 23 participants who completed the intervention arm of the randomized trial, and with the 
2 HT nurses who delivered ACDC; these participants all provided written informed consent for their data to be used in the study, as well as verbal consent for their interviews to be audiorecorded. Five out of 23 participants declined to be interviewed ( $n=4)$ or were lost to follow-up $(n=1)$ at the time that interviews were conducted. Interview questions probed about how intervention components impacted participants and about barriers to engagement with the intervention (Tables 1 and 2). All interviews were transcribed and analyzed using directed content analysis. ${ }^{31}$ Specifically, codes (ie, descriptive labels assigned to text) were designated a priori based on questions about intervention components (eg, "self-monitoring blood glucose"). During initial coding, additional data-derived codes were developed to represent participant characteristics (eg, "handling competing demands") and their perspectives about the intervention's value (eg, "becoming more mindful"). Two experienced health services researchers (NRS and JMG) and a content expert (MJC) coded half of the transcripts individually and then reviewed as a group to achieve consensus through discussion and deliberation. ${ }^{32}$ Codes were refined by another researcher (SMA) using constant comparison technique,

Table I ACDC patient interview guide

Opening questions:

- How do you think your general health has changed since beginning the ACDC program? How has your ability to manage your diabetes changed?

- How was the program for you?

- In what ways was it helpful?

- In what ways was it not helpful?

\section{ACDC home telehealth nurse interaction:}

The telehealth nurse called you at least once every other week to discuss your blood sugars, which we asked you to check at home and send into the VA by telephone.

- What did you think about these calls? Were they helpful or not?

- How did you feel about the frequency of these calls? Were they too often? Not often enough?

- How did you feel about how long these calls took? Were they too long? Too short?

Blood glucose self-monitoring:

Now let us talk a bit about home blood sugar monitoring.

- What was it like for you to measure your blood sugar at home?

- Was there anything difficult about measuring your blood sugars? If so, what?

- Tell me about any changes you have made as the result of measuring your blood sugar at home.

- How did it affect your diet?

- How did it affect your exercise habits?

How did it affect the way you take your medications?

- What other ways did checking your blood sugar levels at home influence your life?

- In the future, how likely would you be to continue monitoring and transmitting blood sugar readings from home if you had the chance? Tell me more about that.

- What would make it easier for you to check and transmit your home blood sugar readings?

- How does at-home blood sugar reading fit with your regular VA care?

- Do you feel that transmitting your home blood sugar readings replaced some of your clinic visits or do you feel that this is an additional piece of your VA care? Tell me more about that.

ACDC diabetes medication management intervention:

The telehealth nurse also called you to give you information about changes to your diabetes medications recommended by our study doctor.

- How did you feel about having your insulin or other medication adjusted by phone?

- How did this affect the way you take your insulin and other medications?

- What did you do with the recommendations? Did you accept them or not? Tell me more.

- How has this affected your relationship with your primary provider?

ACDC self-care behavior support intervention:

The telehealth nurse also reviewed with you lots of information about how to best take care of your diabetes.

- What do you think about the information the nurse shared with you? Did you find it helpful or not? In what ways?

- How did this information affect the way you monitor your sugar, take your insulin, and follow recommendations about diet and exercise?

- What did you do with the recommendations? Did you accept them or not? Tell me more.

- How has this affected your relationship with your primary provider?

Closing questions:

- If we could do anything to change the program we offered you to make it better meet your needs, what would that be?

- Is there anything that you would like to mention that I did not ask about?

Abbreviations: ACDC, Advanced Comprehensive Diabetes Care; VA, Veterans Affairs. 
Table 2 ACDC home telehealth nurse interview guide

\section{Opening questions:}

- In your opinion, what was the most useful part of the program?

- What was challenging?

Patient interaction:

At least once every other week, you spoke with intervention patients by phone to discuss their blood sugars and evaluate medication adherence.

- How receptive were patients to the idea of monitoring their blood sugars and reporting them by phone? Can you tell me more about this?

- How did you feel about the frequency of these calls? Were they too often? Not often enough?

- How did you feel about how long these calls took? Were they too long? Too short?

- What worked well about your interactions with patients with respect to blood sugar monitoring? What did not work well?

- How did patients respond to the recommended blood sugar monitoring? Were they generally adherent or not? Tell me more.

- What do you think are the biggest barriers to adherence to the recommended blood sugar monitoring in this study?

- How was it to assess patients' adherence to their medications during these calls? Do you think that you were able to do this accurately? Tell me more.

- What would you change or improve about the ACDC intervention with respect to blood sugar monitoring and medication adherence evaluation?

ACDC diabetes medication management:

At times, you were asked to relay recommendations regarding diabetes medication adjustments from the study physician to patients.

- How receptive were patients to having their diabetes medications adjusted by phone? Did patients accept the recommendations?

- How long did the process of relaying medication adjustment recommendations take? How could this process be improved?

- What kinds of questions did patients have? How was it for you to answer these?

- How did patients feel about how phone-based medication adjustment might affect their relationship with their primary provider?

- What would you change or improve about the ACDC intervention with respect to the process of diabetes medication adjustment? ACDC self-care behavior support:

You also reviewed information with patients regarding diabetes self-care support.

- How receptive were patients to information regarding diabetes self-care by phone? Did patients accept the recommendations?

- How long did the process of relaying information regarding diabetes self-care take? Was this too long?

- What kinds of questions did patients have? How was it for you to answer these?

- What conversations did you have with patients about how this part of the intervention might affect their relationship with their primary provider? Did they seem concerned? Tell me more.

- What would you change or improve about the ACDC intervention with respect to the process of diabetes self-care support?

Provider interaction:

- In general, what was it like to interact with the study physician and study psychiatrist?

- How did the process whereby you conveyed data to the study providers and received recommendations for medication changes work?

- How available were the study providers for dialog with you when necessary?

- Did you have questions about the rationale behind providers' recommendations? Or did you understand the rationale? Tell me more.

- Did you generally agree with the recommendations? Tell me more.

- How did these interactions generally take place?

- Was there one modality (phone, email, etc) that you used most commonly?

- Was there one that worked the best?

- What would you change or improve about the ACDC intervention with respect to the process of interacting with study providers?

Closing questions:

- If we could do anything else to change the ACDC intervention to make it work better, what would that be?

- Is there anything that you would like to mention that I did not ask about?

Abbreviation: ACDC, Advanced Comprehensive Diabetes Care.

an iterative process of comparing and recoding text as code properties are developed. ${ }^{33,34}$ The final codes were applied to all transcripts (Table 3).

Coded data were then organized in a matrix to identify themes about how participants with similar characteristics and/or outcomes valued program components. Rows represented outcomes of HbAlc change $(<1 \%$ or $\geq 1 \%)$, and columns represented categories of coded data: patient characteristics and program structure.

\section{Results}

\section{Sample characteristics}

Interview participants were 18 men (mean age $=60$ ) who completed ACDC, and the 2 ACDC telehealth nurses, both female. According to inclusion criteria for the ACDC pilot, all participants were identified as having PPDM, defined as $\mathrm{HbAlc}$ of $>9 \%$ for $>1$ year, despite receiving standard clinic-based care. ${ }^{5}$ Participants were categorized according to their HbA1c change during the intervention: $n=4$ were 
Table 3 Derivation of codes applied to ACDC patient interviews

\begin{tabular}{|c|c|c|}
\hline Code grouping & Code & Code origin \\
\hline $\begin{array}{l}\text { Patient } \\
\text { characteristics }\end{array}$ & $\begin{array}{l}\text { - Attitude toward provider } \\
\text { - Balancing multiple chronic } \\
\text { conditions } \\
\text { - Being motivated to change } \\
\text { - Claiming baseline knowledge } \\
\text { - Dealing with depression } \\
\text { - Dealing with stress } \\
\text { - Handling competing demands } \\
\text { - Has consulted with a } \\
\text { - dietitian } \\
\text { - Raving memory issues } \\
\text { - Wanting more information } \\
\text { - Willingness to accept } \\
\text { recommendations }\end{array}$ & Data derived (all) \\
\hline $\begin{array}{l}\text { Program } \\
\text { structure }\end{array}$ & $\begin{array}{l}\text { - Automated telemonitoring } \\
\text { interface } \\
\text { - Integrating with current care } \\
\text { - Medication management } \\
\text { - Self-monitoring and } \\
\text { recording blood glucose } \\
\text { - HT nurse encounters } \\
\text { - Call duration } \\
\text { - Call frequency }\end{array}$ & A priori (all) \\
\hline Program value & $\begin{array}{l}\text { - Acquiring new knowledge } \\
\text { - Applying prior knowledge } \\
\text { - Becoming more mindful } \\
\text { - Changing diet/exercise } \\
\text { - Desiring alternate platform } \\
\text { for SMBG } \\
\text { - Establishing a routine } \\
\text { - Frustrated with interface } \\
\text { - Getting support from } \\
\text { intervention } \\
\text { - Having accountability } \\
\text { - Having increased access to } \\
\text { diabetes care/supplies } \\
\text { - Improving medication } \\
\text { adherence } \\
\text { - Likelihood of continuing to } \\
\text { check and transmit blood } \\
\text { glucose } \\
\text { - Monitoring more frequently } \\
\text { - Seeing results }\end{array}$ & $\begin{array}{l}\text { Data derived } \\
\text { (except "likelihood } \\
\text { of continuing to } \\
\text { check and transmit } \\
\text { blood glucose," } \\
\text { which was a priori) }\end{array}$ \\
\hline
\end{tabular}

Note: A priori represents deductive codes based on program structure. Abbreviations: ACDC, Advanced Comprehensive Diabetes Care; HT, home telehealth; SMBG, self-monitored blood glucose.

classified as "low-change" because their HbA1c improved by $<1 \%$ and $n=14$ were classified as "high-change" because their $\mathrm{HbA} 1 \mathrm{c}$ improved by $\geq 1$. Among low-change participants, $n=2$ completed 3 out of 12 possible encounters with HT nurses, and $n=2$ completed $\geq 6$ out of 12 possible encounters with HT nurses. Among high-change participants, most $(n=13)$ completed $\geq 6$ out of 12 possible encounters.

\section{Findings}

We present aspects of the intervention experience that were common across participants and results for each category of $\mathrm{HbAlc}$ change $(<1 \%$ and $\geq 1 \%)$, using data from both participant and nurse interviews.

\section{Regardless of $\mathrm{HbAlc}$ change, participants reported increased awareness of their diabetes and frustration with the telemonitoring interface}

Self-monitoring more frequently as a result of intervention components led most participants $(\mathrm{n}=15)$ to become aware of fluctuations in their blood glucose. Some $(n=4)$ said they were able to monitor their blood glucose more frequently because study nurses arranged for them to receive selfmonitoring supplies. Participants $(\mathrm{n}=10)$ also described how HT nurse encounters and telemonitoring provided accountability and increased their motivation for self-monitoring. One said,

It is one thing to [...] take your [blood] sugars and record the numbers, but when you are actually entering them into the system $[. .$.$] and having a doctor call you back and say,$ "This day was high; what happened?" [...] It is a little more helpful than just recording them and having no one ask you what is going on.

Nurses also described how they motivated participants: "Some of it is just reinforcing what they already know and encouraging them to take ownership of it."

Another common theme among all participants was the burden imposed by the telemonitoring reporting interface. While daily the telemonitoring component of the intervention provided accountability for their SMBG, many $(n=10)$ indicated that the reporting interface did not fit easily into their daily routines. The VA's telemonitoring system is programmed to deliver follow-up questions and recommendations, based on reported blood glucose values, to all telehealth patients. Participants were frustrated that the length of the telemonitoring calls was extended by computerized recommendations, which they described as repetitive and redundant, and by having to repeat entries when the computer did not correctly record their values. Other participants $(n=5)$ desired more flexibility, for example, to be able to enter $>1$ day's readings at a time rather than telemonitoring daily, or, for those whose work environments did not accommodate regular use of their phones, the ability to report via an alternate device. For example, one participant said, 
When I travel out of the country and I was not able to call in for a couple of weeks, the data was not available. It would be nice if there was a way to do it electronically that would complement the phone service.

For some, the inconvenience and/or stress of using the interface reduced their willingness to engage with the intervention. For example, when describing his attempts to report his blood glucose values, one said:

[The telemonitoring interface] just kept playing, 'Sorry, I did not get that.' And you have to repeat it and repeat it [...] It made me so frustrated that time, that I just hung up the phone and said, 'The hell with it!'

When asked if they would be willing to continue telemonitoring their blood glucose, participants $(\mathrm{n}=5)$ said that they would not be willing if it meant continuing to report their blood glucose through the same telemonitoring system, although most qualified that they would be willing if there were changes to the system (eg, omission of automated recommendations).

\section{Participants whose $\mathrm{HbAlc}$ improved by $<1 \%$} described competing demands that limited their engagement with the intervention

While most low-change participants reported overall satisfaction with the intervention, all $(n=4)$ reported barriers in their lives that affected their capacity to address their diabetes and engage fully with intervention components. Low-change participants $(n=3)$ described health issues such as comorbid illnesses, physical injuries, and medication side effects, which affected their ability or willingness to accept selfmanagement recommendations (eg, to exercise) and adhere to medication adjustments. For example, one participant did not take the medication prescribed by the study physician because he said that they made him feel sick. Another described shoulder pain that prevented him from working for 3 months, with resultant high blood glucose values that he attributed to stress. And one described how "depression" hindered his self-monitoring, saying,

Sometimes I got the grit that I can do it, and a couple of days or later on that afternoon I may be in a different mood $[\ldots]$ and I do not give a damn.

Low-change participants $(\mathrm{n}=3)$ also said that their daily commitments, including work and family obligations, did not accommodate intervention components. When referring to the telemonitoring process, one participant said:

That [telemonitoring interface] would keep you on the phone for fifteen to twenty minutes. I have not got that time.
I got three kids, a wife, and we are a very busy family. Plus

I work twelve hour shifts at work.

Another said that his irregular schedule made it difficult to perform important self-care activities such as eating and taking medications at specific times; he also said that he frequently forgot to bring his mobile phone with him during the day, rendering him unable to participate in telemonitoring.

Nurse feedback echoed comments by some low-change participants about their difficulty participating in intervention components. One nurse said:

I had one patient $[\ldots]$ who actually works two jobs and he is gone from like 7-3 and 3-midnight. I mean, there was almost no time that you could ever call this man. I think I got him on the phone twice.

She also reported seeing a correlation between participation in nurse HT encounters and participation in the telemonitoring component of the intervention, saying: "The same ones that we struggled trying to contact, they also struggled with reporting."

\section{Participants whose HbAlc improved by $\geq 1 \%$ described how participation in the intervention bolstered their capacity to manage their diabetes}

Some high-change participants $(\mathrm{n}=9)$ reported similar challenges to participation as low-change participants, including comorbid injury and illness, work schedules that conflicted with telemonitoring, and family responsibilities. Despite these challenges, high-change participants reported that they engaged with intervention components and described how the intervention supported them in ways that bolstered their capacity to self-manage.

Participants $(n=8)$ who achieved the highest HbAlc change described improved diabetes control when the study physician made multiple adjustments to their medications and they adhered to these adjustments. Some $(\mathrm{n}=3)$ appreciated that the study physician accommodated their medication preferences, such as wanting to avoid injectable insulin, and attributed improved adherence to finding their medication regimens tolerable. For example, one said:

They give me medication that would work for me. It is much easier to achieve your goal if you can trust and depend on what the doctors give you [...] Honestly, I think [the medication] was the biggest factor in me being able to stay with the program.

High-change participants followed recommendations even in rare cases when they experienced an initial suboptimal 
reaction to a medication adjustment. For example, one participant who experienced a brief period of hypoglycemia after a medication adjustment said,

I have a family history of diabetes. I want to keep it under control as much as possible. I followed [medication recommendations] exactly the way they give them to me.

High-change participants $(n=7)$ also described how personalized education from the HT nurses about their diet and medications helped them establish new routines that led to better diabetes control. One said:

The biggest help was educating more about what to eat, and when to take my pills. Because you know I was taking my pills randomly [...] it helped me to know that you are supposed to take a pill, then after you take a pill, you eat 30 minutes after you take the pill.

Participants $(n=4)$ also described how they developed new eating habits after their HT nurse arranged individualized nutrition counseling with a dietitian. Nurses echoed the importance of personalized education in forming new selfmanagement routines and attributed participants' positive outcomes to more frequent encounters. One said,

I have heard other Veterans not in [ACDC] say, "Well it is taking me a week or two for [HT providers] to tell me what to do with my medications." With [ACDC] [...] as soon as [study physician] would tell me what to do, I would call them and we would be changing medications frequently in very short time periods, and it wound up to make an even more of an impact [...] than I had seen previously in the diabetic population that was just in home telehealth.

Nurses stated that the frequency of their contact with ACDC participants allowed them to form a relationship that differed from their typical telehealth nursing encounters, which only occurred when a patient's reported values were dangerously high or low.

High-change participants $(n=6)$ described that their motivation to manage their diabetes increased as they received support from intervention components. For example, one described how the HT nurse helped him prioritize his diabetes self-care:

When I first started the program I was a caretaker for my disabled mother and I was paying more attention to taking care of her than I was myself [...] I learned [...] with talking with [HT nurse] how important it was for me to take care of myself as well.

Others $(n=5)$ said that they were motivated to improve their diabetes control prior to starting the intervention and thus were more receptive to intervention components. For example, one said: "Well, I was just kind of feeling like crap all the time [...] So, I was looking for a change and I was ready for a change."

\section{Discussion}

The ACDC intervention was designed to comprehensively address factors contributing to PPDM by combining telemonitoring, self-management support, and medication management. Critically, ACDC sought to leverage existing VA telemedicine infrastructure to promote practical intervention delivery and potentially facilitate eventual implementation. Feedback from participants generally indicated that this combination of strategies supported them in improving $\mathrm{HbA1c}$ through enhanced awareness of blood glucose fluctuation, provision of support for developing self-management routines, and frequent medication management, even though there was widespread frustration with the telemedicine interface. Differences were noted between participants with higher versus lower HbA1c improvement, in that participants with $<1 \%$ improvement reported that competing demands interfered with their engagement, and participants with $\geq 1 \%$ improvement reported increased capacity to self-manage as a result of engaging with the intervention.

Prior studies have elucidated mechanisms by which the three ACDC intervention components are effective: telemonitoring has been described as a cue to action and source of accountability for SMBG that leads to increased awareness of diabetes control; ${ }^{20,23,26,35}$ self-management support has been described as a source of accountability and social support; ${ }^{21,25,28,36}$ and more frequent medication management has been shown to improve clinical outcomes and patientprovider relationships. ${ }^{24,27,37-39}$ ACDC participants' description of their interactions with intervention components supported these proposed mechanisms of effectiveness. ACDC builds on existing knowledge through two innovations: the comprehensive nature of the intervention and the use of existing telemedicine infrastructure to deliver the intervention.

\section{Treating a complex disease with a comprehensive intervention}

By focusing on PPDM, we deliberately sought out the highest risk diabetes patients in VA and targeted them with an intensive, comprehensive telemedicine intervention. Because patients with PPDM contribute disproportionately to the nationwide diabetes burden, ${ }^{6,7}$ addressing PPDM with a high-intensity intervention like ACDC may represent an appropriate matching of resources to clinical need. Our 
results suggest that it may be the combination of intervention strategies, rather than individual strategies, which had the greatest effect on outcomes. High-change participants indicated that telemonitoring and HT nurse encounters prompted them to self-monitor more frequently, which increased their awareness of blood glucose fluctuations. Participants also received regular medication management based on the blood glucose values they reported. As they accepted medication adjustments and made lifestyle changes in response to SMBG, it is possible that patients' improved diabetes control enhanced their motivation for self-management, leading to further positive behavior change. Thus, intervention components may have interacted to initiate a positive feedback loop wherein participants' engagement with the intervention led to positive clinical outcomes and rendered them better able and more motivated to sustain changes. ${ }^{17}$

\section{Capitalizing on existing infrastructure to facilitate practical delivery}

To foster comprehensive telemedicine-based management as a much-needed alternative to insufficiently effective clinicbased care for PPDM, we designed ACDC for practical delivery using existing VA infrastructure. There is a clear clinical need for alternative approaches when patients do not respond to clinic-based measures; but to our knowledge, neither VA nor any other system has addressed this need in practice. By leveraging VA's investment in telemedicine, ACDC has potential for eventual widespread implementation and thus ample promise to widely impact Veterans with PPDM.

Of note, we found that our use of existing VA telemonitoring infrastructure was a source of frustration for many patients. The use of telemedicine modalities is intended to alleviate treatment burden, but telemonitoring interfaces have been cited as barriers to participation and impediments to satisfaction in many telemedicine studies. ${ }^{24,26,35,36}$ This is problematic, because the fit of telemedicine innovations into patients' lives is critical to the success and sustainability of interventions that employ them. ${ }^{26}$ In ACDC, the limitations of the VA's telemonitoring interface burdened some participants to the point of impeding participation.

Future work should focus on optimizing telemonitoring infrastructure to minimize burden on participants, thus facilitating engagement in self-management activities. However, the potential advantages of innovative telemonitoring technology that is more user-friendly, but less widely available, must be weighed against the potential for widespread implementation. ${ }^{28,29}$ Despite frustration with the VA's existing telemonitoring interface, most ACDC participants still achieved significant $\mathrm{HbA} 1 \mathrm{c}$ change using the interface as part of a comprehensive intervention; thus, given current VA infrastructure, telephone-based care strikes a reasonable balance between effectiveness, reach, and potential scalability. As technology evolves, and VA develops infrastructure to deliver alternatives to phone-based care delivery, ACDC's telemonitoring platform will evolve accordingly.

\section{Minimizing treatment burden when intervening on complex diseases}

Shippee et $\mathrm{al}^{17}$ posit that for patients with complex conditions such as PPDM, engagement with health care is affected by the balance between their workload (ie, demands on their time and energy such as employment, self-care activities such as telemonitoring of SMBG) and their capacity to address their demands (ie, physical and mental ability, resources, and readiness). A patient's disease(s) can limit his capacity, and treatments to address disease(s) can increase his workload. ${ }^{17,18}$ Our data support the notion that for low-change participants, burden imposed by the intervention (ie, difficulty using the telemonitoring interface), combined with other demands in their lives, contributed to imbalance between their workload and their capacity to manage their diabetes; consequently, they were unable to engage with intervention components.

In contrast, feedback from high-change participants suggests that, while they may have also experienced increased burden as a result of intervention components, their capacity to manage this burden allowed them to engage with the intervention; intervention components then supported them in improving clinical outcomes, which further increased their capacity to self-manage. Additionally, our data suggest that some high-change participants were motivated to change prior to initiating the intervention. It is possible that for these participants, recognition of their disease severity and an increased readiness to change at baseline offset the effects of other factors in their lives that might have otherwise limited their engagement.

Ultimately, PPDM is a complex condition that requires comprehensive treatment. However, increasing the intensity of treatment, by definition, imposes additional burden on patients who may already have reduced capacity to manage their diabetes. ${ }^{17,18}$ Therefore, interventions targeting PPDM may be most successful if they include assessment of workload and capacity barriers prior to enrolling patients, and provision of capacity-bolstering support to offset treatment burden. Because social support and socioeconomic/cultural factors can also influence participants' capacity to engage 
with interventions like ACDC, ${ }^{12,13,16}$ future work should focus on further tailoring intervention content and delivery based on participants' level of social support, socioeconomic status, and cultural background.

\section{Conclusion}

We found that a comprehensive telehealth intervention that includes telemonitoring of SMBG, self-management support, and personalized medication management helped many participants with PPDM improve their diabetes control. However, intervention factors (eg, ease-of-use of telemonitoring technology) and individual factors (eg, the presence of competing life demands) affected some participants' engagement with the intervention. Differences in engagement may reflect differing capacity to manage treatment burden.

This approach is a promising model for addressing PPDM within VA and beyond. Within VA, the ability to leverage existing clinical infrastructure improves the feasibility of delivering this successful pilot intervention on a larger scale. Future work should focus on optimizing infrastructure to minimize the tension caused by using technology that promotes the scope of intervention delivery but may limit intervention engagement, and on developing alternate technology interfaces that are more satisfactory to participants (eg, mobile applications). Organizations implementing telemedicine interventions for PPDM should consider bundling strategies such as telemonitoring, regular self-management support, and frequent medication management, which comprehensively address factors that contribute to poor diabetes control. Additionally, our findings suggest that since patients with PPDM have a high clinical burden and are likely to have additional life demands, interventions targeted at this population may be more successful if they assess workload and capacity factors that may impede engagement. Patients who are identified as having a potential imbalance between workload and capacity may require more intensive support prior to, or during, interventions.

\section{Acknowledgments}

This work was supported by a grant from the US Department of Veterans Affairs, Veterans Health Administration, Health Services Research \& Development (Diabetes QUERI RRP 12-458), and the Center for Health Services Research in Primary Care (COIN CIN 13-410). Portions of these data were presented at the American Diabetes Association Scientific Sessions, held in Boston, Massachusetts, June 8, 2015, and the Veterans Affairs Health Services Research and Development National Meeting, held in Philadelphia, PA,
July 10, 2015. The views expressed in this article are those of the authors and do not necessarily reflect the position of the Department of Veterans Affairs or Duke University.

\section{Disclosure}

MJC is supported by a Career Development Award from VHA Health Services Research and Development (CDA 13-261). The other authors report no conflicts of interest in this work.

\section{References}

1. Stratton IM, Adler AI, Neil HA, et al. Association of glycaemia with macrovascular and microvascular complications of type 2 diabetes (UKPDS 35): prospective observational study. BMJ. 2000;321(7258): 405-412.

2. GilmerTP, O'Connor PJ, Manning WG, Rush WA. The cost to health plans of poor glycemic control. Diabetes Care. 1997;20(12):1847-1853.

3. McBrien KA, Manns BJ, Chui B, et al. Health care costs in people with diabetes and their association with glycemic control and kidney function. Diabetes Care. 2013;36(5):1172-1180.

4. Ali MK, McKeever Bullard K, Imperatore G, Barker L, Gregg EW. Characteristics associated with poor glycemic control among adults with selfreported diagnosed diabetes - National Health and Nutrition Examination Survey, United States, 2007-2010. MMWR Suppl. 2012;61(2):32-37.

5. Crowley MJ, Edelman D, McAndrew AT, et al. Practical telemedicine for veterans with persistently poor diabetes control: a randomized pilot trial. Telemed J E Health. 2016;22(5):376-384.

6. Ali MK, Bullard KM, Gregg EW. Achievement of goals in U.S. Diabetes Care, 1999-2010. N Engl J Med. 2013;369(3):287-288.

7. Riddle MC, Ambrosius WT, Brillon DJ, et al. Epidemiologic relationships between $\mathrm{A} 1 \mathrm{C}$ and all-cause mortality during a median 3.4-year follow-up of glycemic treatment in the ACCORD trial. Diabetes Care. 2010; 33(5):983-990.

8. Crowley MJ, Holleman R, Klamerus ML, Bosworth HB, Edelman D, Heisler M. Factors associated with persistent poorly controlled diabetes mellitus: clues to improving management in patients with resistant poor control. Chronic Illn. 2014;10(4):291-302.

9. Richardson C, Kerr EA, Krein SL, et al. Diabetes QUERI 2011 Strategic Plan. Washington DC: Veterans Affairs Center for Clinical Management Research; 2011. Available from: http://ibrarian. net/navon/paper/Diabetes_QUERI_Center_2011_Strategic_Plan. pdf?paperid=20519140. Accessed January 23, 2017.

10. Morrison F, Shubina M, Turchin A. Encounter frequency and serum glucose level, blood pressure, and cholesterol level control in patients with diabetes mellitus. Arch Intern Med. 2011;171(17):1542-1550.

11. Pimouguet C, Le Goff M, Thiebaut R, Dartigues JF, Helmer C. Effectiveness of disease-management programs for improving diabetes care: a meta-analysis. Can Med Assoc J. 2011;183(2): E115-E127.

12. Bailey CJ, Kodack M. Patient adherence to medication requirements for therapy of type 2 diabetes. Int J Clin Pract. 2011;65(3):314-322.

13. Ahola AJ, Groop PH. Barriers to self-management of diabetes. Diabet Med. 2013;30(4):413-420.

14. Nelson KM, McFarland L, Reiber G. Factors influencing disease selfmanagement among veterans with diabetes and poor glycemic control. J Gen Intern Med. 2007;22(4):442-447.

15. Fisher WA, Kohut T, Schachner H, Stenger P. Understanding selfmonitoring of blood glucose among individuals with type 1 and type 2 diabetes: an information-motivation-behavioral skills analysis. Diabetes Educ. 2011;37(1):85-94.

16. Gonzalez JS, Tanenbaum ML, Commissariat PV. Psychosocial factors in medication adherence and diabetes self-management: implications for research and practice. Am Psychol. 2016;71(7):539-551. 
17. Shippee ND, Shah ND, May CR, Mair FS, Montori VM. Cumulative complexity: a functional, patient-centered model of patient complexity can improve research and practice. J Clin Epidemiol. 2012;65(10): 1041-1051.

18. May C, Montori VM, Mair FS. We need minimally disruptive medicine. BMJ. 2009;339:b2803.

19. American Telemedicine Association. What is Telemedicine? 2016. Available from: http://www.americantelemed.org/about/telehealthfaqs-. Accessed July 15, 2016.

20. Ontario HQ. Home telemonitoring for type 2 diabetes: an evidencebased analysis. Ont Health Technol Assess Ser. 2009;9(24):1-38.

21. Ontario HQ. Behavioural interventions for type 2 diabetes: an evidencebased analysis. Ont Health Technol Assess Ser. 2009;9(21):1-45.

22. Flodgren G, Rachas A, Farmer AJ, Inzitari M, Shepperd S. Interactive telemedicine: effects on professional practice and health care outcomes. Cochrane Database Syst Rev. 2015;9:Cd002098.

23. Pare G, Moqadem K, Pineau G, St-Hilaire C. Clinical effects of home telemonitoring in the context of diabetes, asthma, heart failure and hypertension: a systematic review. J Med Internet Res. 2010;12(2):e21.

24. Klug C, Bonin K, Bultemeier N, et al. Integrating telehealth technology into a clinical pharmacy telephonic diabetes management program. J Diabetes Sci Technol. 2011;5(5):1238-1245.

25. Nundy S, Mishra A, Hogan P, Lee SM, Solomon MC, Peek ME. How do mobile phone diabetes programs drive behavior change? Evidence from a mixed methods observational cohort study. Diabetes Educ. 2014; 40(6):806-819.

26. Vassilev I, Rowsell A, Pope C, et al. Assessing the implementability of telehealth interventions for self-management support: a realist review. Implement Sci. 2015;10:59.

27. Marzec LN, Maddox TM. Medication adherence in patients with diabetes and dyslipidemia: associated factors and strategies for improvement. Curr Cardiol Rep. 2013;15(11):418.

28. Bodenheimer T, Lorig K, Holman H, Grumbach K. Patient selfmanagement of chronic disease in primary care. JAMA. 2002;288(19): 2469-2475.
29. Glasgow RE, Lichtenstein E, Marcus AC. Why don't we see more translation of health promotion research to practice? Rethinking the efficacy-to-effectiveness transition. Am J Public Health. 2003;93(8): 1261-1267.

30. Milat AJ, King L, Bauman AE, Redman S. The concept of scalability: increasing the scale and potential adoption of health promotion interventions into policy and practice. Health Promot Int. 2013;28(3): 285-298.

31. Hsieh HF, Shannon SE. Three approaches to qualitative content analysis. Qual Health Res. 2005;15(9):1277-1288.

32. Hill CEKS, Thompson BJ, Williams EN, Hess SA, Ladany N. Consensual qualitative research: an update. J Couns Psychol. 2005;52(2): 196-205.

33. Glaser BG, Strauss AL. The Discovery of Grounded Theory: Strategies for Qualitative Research. New York: Aldine De Gruyter; 1967.

34. Charmaz K. Constructing Grounded Theory: A Practical Guide Through Qualitative Analysis. First ed. London: Sage Publications Ltd; 2006.

35. Tatara N, Arsand E, Skrovseth SO, Hartvigsen G. Long-term engagement with a mobile self-management system for people with type 2 diabetes. JMIR MHealth UHealth. 2013;1(1):e1.

36. Trief PM, Sandberg J, Izquierdo R, et al. Diabetes management assisted by telemedicine: patient perspectives. Telemed J E Health. 2008;14(7):647-655.

37. McMahon GT, Gomes HE, Hickson Hohne S, Hu TM, Levine BA, Conlin PR. Web-based care management in patients with poorly controlled diabetes. Diabetes Care. 2005;28(7):1624-1629.

38. Brown NN, Carrara BE, Watts SA, Lucatorto MA. RN diabetes virtual case management: a new model for providing chronic care management. Nurs Adm Q. 2016;40(1):60-67.

39. Rosenthal ES, Bashan E, Herman WH, Hodish I. The effort required to achieve and maintain optimal glycemic control. J Diabetes Complications. 2011;25(5):283-288.
Patient Preference and Adherence

\section{Publish your work in this journal}

Patient Preference and Adherence is an international, peer-reviewed, open access journal that focuses on the growing importance of patient preference and adherence throughout the therapeutic continuum. Patient satisfaction, acceptability, quality of life, compliance, persistence and their role in developing new therapeutic modalities and compounds to optimize

\section{Dovepress}

clinical outcomes for existing disease states are major areas of interest for the journal. This journal has been accepted for indexing on PubMed Central. The manuscript management system is completely online and includes a very quick and fair peer-review system, which is all easy to use. Visit http://www. dovepress.com/testimonials.php to read real quotes from published authors. 